\title{
Absence of cerebrospinal fluid antineuronal antibodies in schizophrenia spectrum disorders - CORRIGENDUM
}

Tatiana Oviedo-Salcedo, Lot de Witte, Tania Kümpfel, René S. Kahn, Peter Falkai, Peter Eichhorn, Jurjen Luykx and Alkomiet Hasan

\section{Summary}

This notice describes a correction to the above mentioned paper.

\section{Copyright and usage}

(c) The Royal College of Psychiatrists 2018. https://doi.org/10.1192/bjp.2018.24, Published by Cambridge University Press, 28 March, 2018.

In the reference list, number 11 is stated incorrectly. The correct reference for 11 is:

Dalmau J, Lancaster E, Martinez-Hernandez E, Rosenfeld MR, Balice-Gordon R. Clinical experience and laboratory investigations in patients with anti-NMDAR encephalitis. The Lancet Neurology 2011; 10(1):63-74.

The authors regret the error.
Tatiana Oviedo-Salcedo, Lot de Witte, Tania Kümpfel, René S. Kahn, Peter Falkai, Peter Eichhorn, Jurjen Luykx, Alkomiet Hasan

\section{Reference}

Oviedo-Salcedo T, De Witte L, Kümpfel T, Kahn R, Falkai P, Eichhorn P, Luykx J, Hasan A. Absence of cerebrospinal fluid antineuronal antibodies in schizophrenia spectrum disorders. The British Journal of Psychiatry 2018; 1-3. doi:10.1192/bjp.2018.24. 